\title{
Turning the tide: mutually evasive ebb- and flood-dominant channels and bars in an experimental estuary
}

\author{
M. G. Kleinhans, T.M. van Rosmalen, C. Roosendaal, and M. van der Vegt \\ Faculty of Geosciences, Universiteit Utrecht, Utrecht, the Netherlands \\ Correspondence to: M. G. Kleinhans (m.g.kleinhans@uu.nl)
}

Received: 17 October 2013 - Revised: 25 November 2013 - Accepted: 28 November 2013 - Published: 1 April 2014

\begin{abstract}
Tidal bars in estuaries are poorly understood compared to fluvial bars. There is limited theory that predicts tidal bar dimensions. Moreover, where fluvial channels bifurcate around bars, tidal channels around bars often develop either flood- or ebb-dominance which remains unexplained. Tidal experiments are rare and have not reproduced these phenomena to date because these are usually highly ebb-dominated. Here we report preliminary experiments in a novel setup that produce tidal bars from an initial situation with flat bed and exactly symmetrical ebb- and flood-related sediment mobility. The bars initially develop in a saw-tooth pattern with mutually evasive ebb- and flood channels. The tips of the bars evolve further into lobate bars built up from sediment from excavating channels. The bar tips form steep and high obstructions for the opposing flow direction, which then diverges into evasive channels. The bars develop into a diamond shape as channels bifurcate and deepen around it. We further found that bar height correlates well to bar width but not with bar length, whilst bar length correlates well to the tidal excursion length. As the experiment progressed scour holes developed that affected the channel and bar pattern and are considered artifacts of the experiment. We conclude that the mutually evasive ebb- and flood-dominance of channels emerges simultaneously with the onset of tidal bar formation, and that these preliminary data confirm results from linear stability analyses. The results are proof of principle for the novel experimental setup for tidal systems.
\end{abstract}

\section{Introduction}

Tidal bars in estuaries are poorly understood whereas for rivers there are adequate empirical and theoretical predictors for bar length, bar mode (or number of bars in the cross- section) and meander wavelength (e.g. Kleinhans and van den Berg, 2011). For the tidal environment we lack adequate empirical bar descriptions (Dalrymple and Rhodes, 1995) but observations show that bar shape differs significantly between estuaries (e.g. Gironde, Columbia River, Moreton Bay, Cobequid Bay). The few theoretical predictors for initially formed tidal bars disagree on fundamental causality, namely whether bar mode and bar length depend on the basin widthto-depth ratio (Seminara and Tubino, 2001) or on the tidal excursion length (defined as characteristic flow velocity divided by tidal period) (Schramkowski et al., 2002).

Channels that surround the tidal bars are also fundamentally different from those in rivers. Field observations show that specific ebb- and flood-dominated channels emerge (van Veen, 1950). Such channels often terminate in lobate bars or shoals with similarities with chute channels and bars. However, the orientation and morphodynamics depend strongly on the direction of the current, i.e. the phase of the tide. Such mutually evasive ebb- or flood-dominated channels also emerged in numerical modelling (Hibma et al., 2003) but why the phenomenon arises remains unclear.

Our objective is to understand formative processes and shapes of tidal bars in sandy estuaries. We employ an underused method in coastal geomorphology: experiments. This work is complementary to our earlier work in a tilting flume with an asymmetrical initial morphology and one open boundary that resulted in short tidal wadden systems with ebb deltas (Kleinhans et al., 2012).

\section{Novel method for tidal experiments}

Experiments to simulate tidal systems are rare compared to fluvial experiments or simulation by numerical models. In the few publications that report morphodynamic experiments 
Table 1. Experimental settings. The delay between tilting phases was $2 \mathrm{~s}$. The flume was $1.3 \mathrm{~m}$ wide in Exp. 2 and narrowed to $1.0 \mathrm{~m}$ wide in all other experiments.

\begin{tabular}{llccccccc}
\hline Exp. & tidal “energy" & $\begin{array}{c}\text { Slope } \\
\mathrm{mm} \mathrm{m}^{-1}\end{array}$ & $\begin{array}{c}\text { Period } \\
\mathrm{s}\end{array}$ & $\begin{array}{c}\text { Tilt speed } \\
\mathrm{mm} \mathrm{min}^{-1}\end{array}$ & $\begin{array}{c}\text { Depth } \\
\mathrm{mm}\end{array}$ & $\begin{array}{c}\text { Duration } \\
\mathrm{h}\end{array}$ & Bar shape & Fig. \\
\hline 1 & low & 2.5 & 64 & 16 & 5 & 68 & V-shaped & 2 \\
2 & moderate & 3.1 & 90 & 16 & 3 & 80 & transitional & 3 \\
3 & high & 3.1 & 90 & 14 & 5 & 75 & transitional & 4 \\
4 & high & 2.8 & 71.5 & 16 & 5 & 70 & diamond-shaped & 5 \\
B & fluvial & 2.5 & - & 16 & 5 & 4 & braiding & \\
\hline
\end{tabular}

with tides (e.g. Reynolds, 1889; Mayor-Mora, 1977; Tambroni et al., 2005; Stefanon et al., 2010) the system is reported to evolve towards a static situation with only minor sediment transport. The reason is that water depth is reduced much more than sediment size in the flume compared to natural systems, so that a much larger water surface slope than in nature is needed to drive the sediment transport. However, such a large ebb and flood water surface slope would be very different from the bed slope, which would cause unnatural differences in tidal amplitude, phase and sediment mobility along the flume. Furthermore, in the case of systems that deepen seawards, the exaggerated bed slope of an experiment would strongly promote ebb-related transport and reduce or prevent flood-related sediment transport. Thus the system becomes entirely ebb-dominated until so much sediment is exported that the threshold for motion is no longer exceeded and a static equilibrium results, in contrast to the dynamic equilibrium observed in nature.

To mitigate the sediment mobility problem the entire flume is periodically tilted over the short axis. By tilting we exaggerate the flume slope to compensate for the much smaller water depth and still have sediment transport. The advantage of this novel method is that the bed surface slopes in downstream direction both during flood and ebb phase, resulting in significant transport and morphological change in the flood phase as well as the ebb phase (Kleinhans et al., 2012). This avoids the scaling effect that hampered all tidal experiments in the past. The resulting flow is close to the rigid lid assumption of most theories (e.g. Seminara and Tubino, 2001; Schramkowski et al., 2002).

We experimentally created tidal bars in a short reach within a tidal channel. Here we present four representative experiments (Table 1) but many more were performed. Conditions were similar as in our experiments of short tidal basins with self-formed channel networks and ebb deltas (Kleinhans et al., 2012). The flume, $3.5 \mathrm{~m}$ by $1.3 \mathrm{~m}$, had initially flat bed raised above the sea floor (Fig. 1). Sediment was poorly sorted blasting polymer between $0.3-$ $1.1 \mathrm{~mm}$ with $\mathrm{D} 50=0.62 \mathrm{~mm}$ and $\mathrm{D} 90=1.1 \mathrm{~mm}$ and density $1200 \mathrm{~kg} \mathrm{~m}^{-3}$ to enhance sediment mobility. The poor sorting was chosen because this reduces the occurrence of unrealistic scour holes (Van Dijk et al., 2012). For control we also did
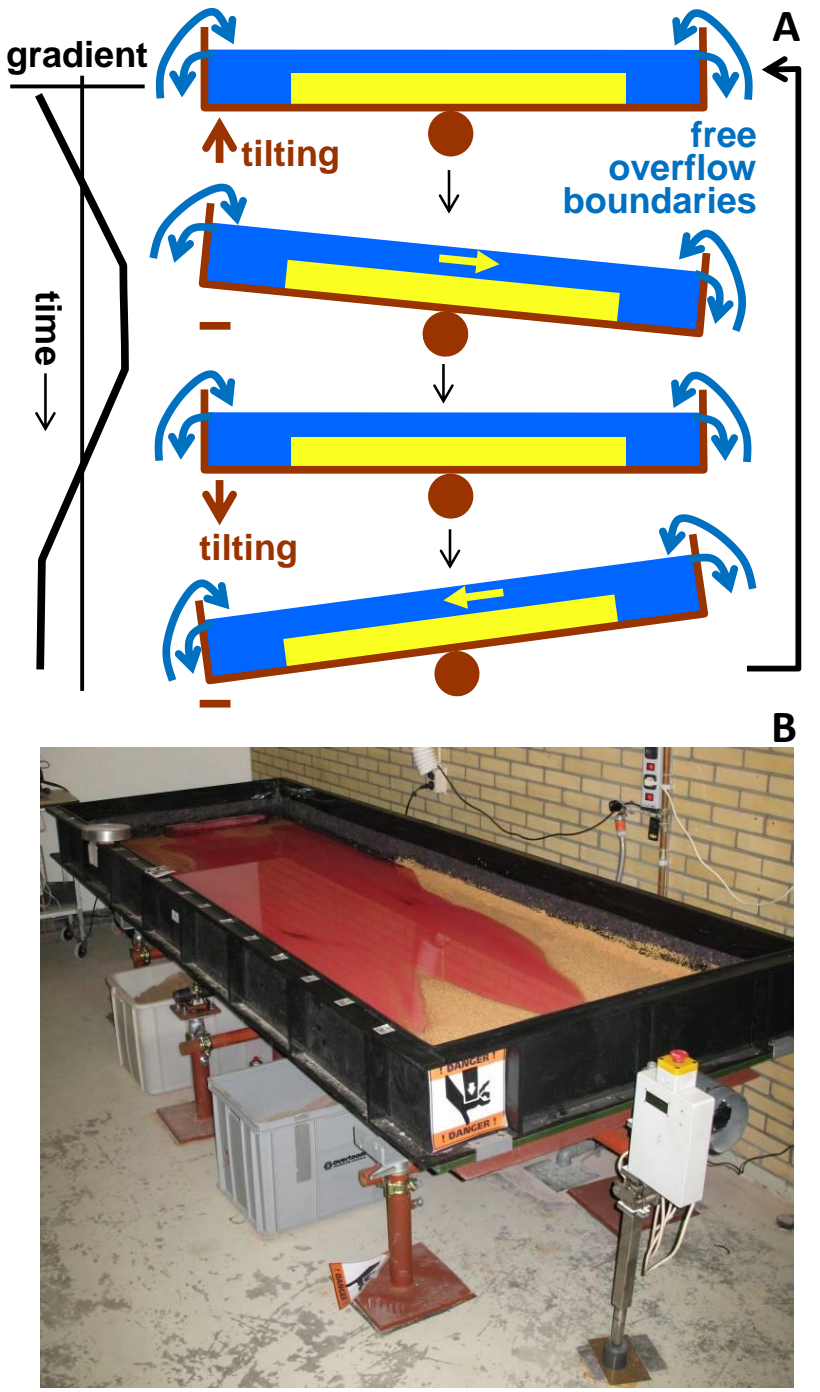

Fig. 1. Tilting flume to create bidirectional tidal currents and sediment transport. A. Schematic drawing showing the principle. B. Photograph of the flume in a similar experiment. The flume width is $1.3 \mathrm{~m}$. Amplitude, constant tilting speed and delay between alternating tilting directions are adjustable (leg with stepping motor in bottom right). Fixed free overflow boundaries ensure a constant head. 
unidirectional flow experiments without tilting but otherwise similar conditions (Table 1).

Tidal (tilting) period was of the order of a minute with a slope of a few $\mathrm{mm} \mathrm{m}^{-1}$ and initial basin water depth $5 \mathrm{~mm}$ (Table 1). The tilting speed was constant during tilting and zero for a set time before tilting in the opposite direction commenced (Fig. 1). Although this is only a rough approximation of harmonic tilting the flow in the flume is clearly smoothly harmonic without shocks. Future PIV measurements may quantify the precise tidal flow but visual observation of floats strongly suggests a simple harmonic variation of flow velocity. Different combinations of tilting speed and period could result in similar energy and a calculation of tidal energy from the tilt settings is not straightforward. The experiments were therefore ordered as low energy, moderate energy and high energy on the basis of the intensity of sediment transport and morphological change per tidal cycle observed visually.

The morphological development was recorded with digital overhead cameras synchronised with the tilting at every maximum tilt and horizontal position. Blue dye added to the water indicates depth in the images. A fringe projector and calibrated digital camera system ("Z-snapper") recorded dry bed elevation of selected times at sub-mm resolution. Bars were defined by zero-crossings on bed elevation with initial elevation subtracted.

\section{Results}

Typical flow velocity measured with surface tracers on average ranged between $0.10-0.20 \mathrm{~m} \mathrm{~s}^{-1}$ for different experiments. Initial depth was $5 \mathrm{~mm}$ and typical depths in channels were $10-20 \mathrm{~mm}$. In the channels both velocity and depth were not more than a factor of two larger. This means that flow was on average transitional to turbulent with Reynolds numbers of about 1000 up to 4000 in the channels, and subcritical flow with Froude numbers below unity. The tidal excursion length, here estimated as the maximum tidal surface flow velocity multiplied with half the tidal period, is the same order of magnitude as the flume length, which means that the experiments represent a short reach within an estuary. Sediment mobility was intermediate between bed-load and suspended load-dominated with Shields numbers of about 0.1 .

All tidal experiments had similar bar initiation processes and patterns (Figs. 2-5). Bars initiated everywhere in the flume from minor perturbations in the flat bed. In less than an hour the bars and channels became asymmetrical and migrated in only one phase of the tide. This means that mutually evasive ebb- or flood-dominated channels evolved without any asymmetry or forcing in the system. The initial development from perturbations was slow but once channels had formed the flow became more focussed so that the energy per unit active width increased.
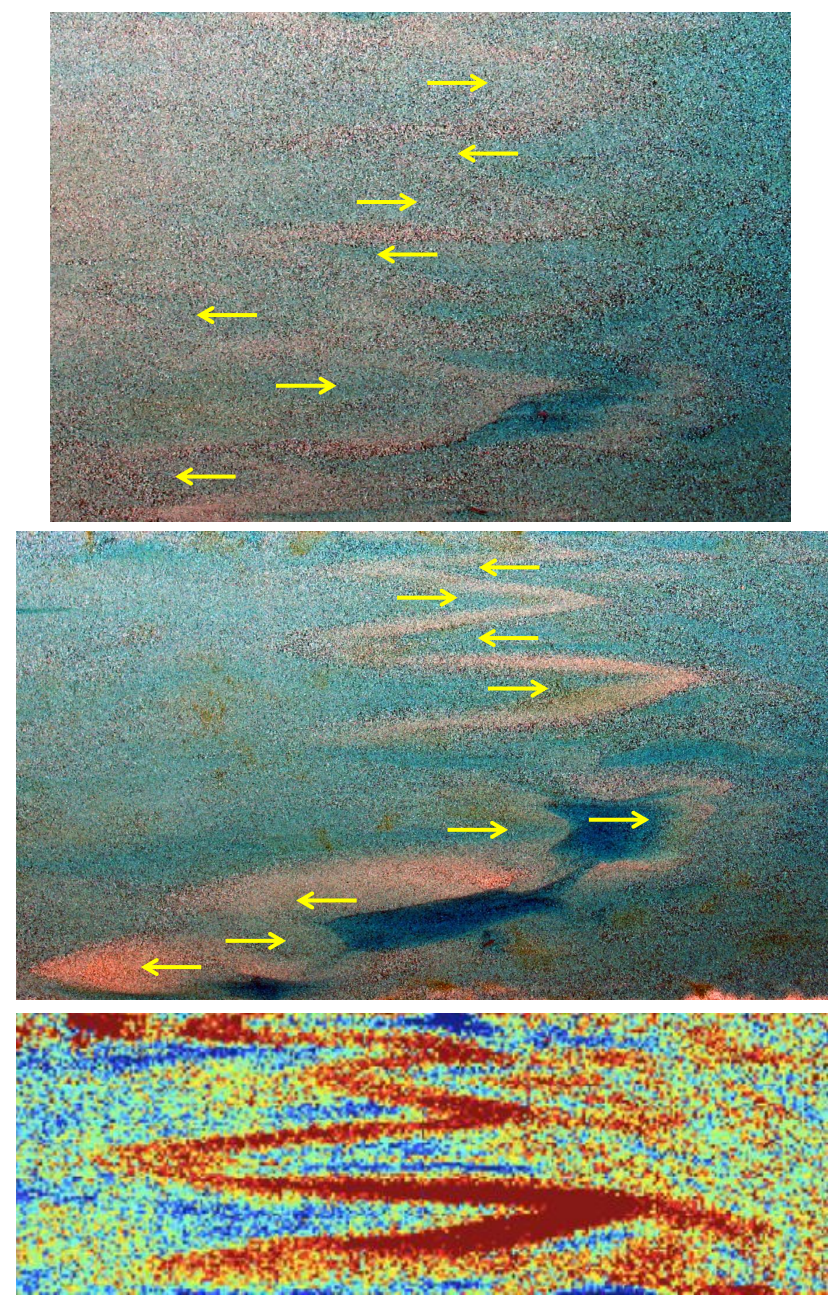

Fig. 2. Top view on V-shaped tidal bars early and late in low-energy experiment 1 after about 100 and 1000 tidal cycles. Blue colour indicates water depth and arrows indicate dominant tidal flow direction. Bottom panel shows DEM of V-shaped bars with red and blue colours $1.5 \mathrm{~mm}$ above and below initial bed level.

The smallest bars that formed initially in all experiments and remained present in the low-energy experiments were sharply V-shaped, connected in a saw-tooth pattern, with points alternating in the dominant tidal flow direction (top panels in Figs. 2-4). Apparently the points of the bars had higher hydraulic resistance in the opposite flow direction, because when the tidal current reversed the flow did not concentrate in the same channel but in the flanking channels.

As channels excavated in the more energetic experiments, the sediment was transported to form a diamond-shaped bar or sometimes lobate bar (Figs. 3-5). In turn, the flow of the opposite tidal phase split around these bars to form new channels flanking the bars. These channels also formed lobate bars in the opposite direction.

On the initially lobate bars flow diverged in a manner similar to chute bars in rivers or mouth bars in deltas. The 

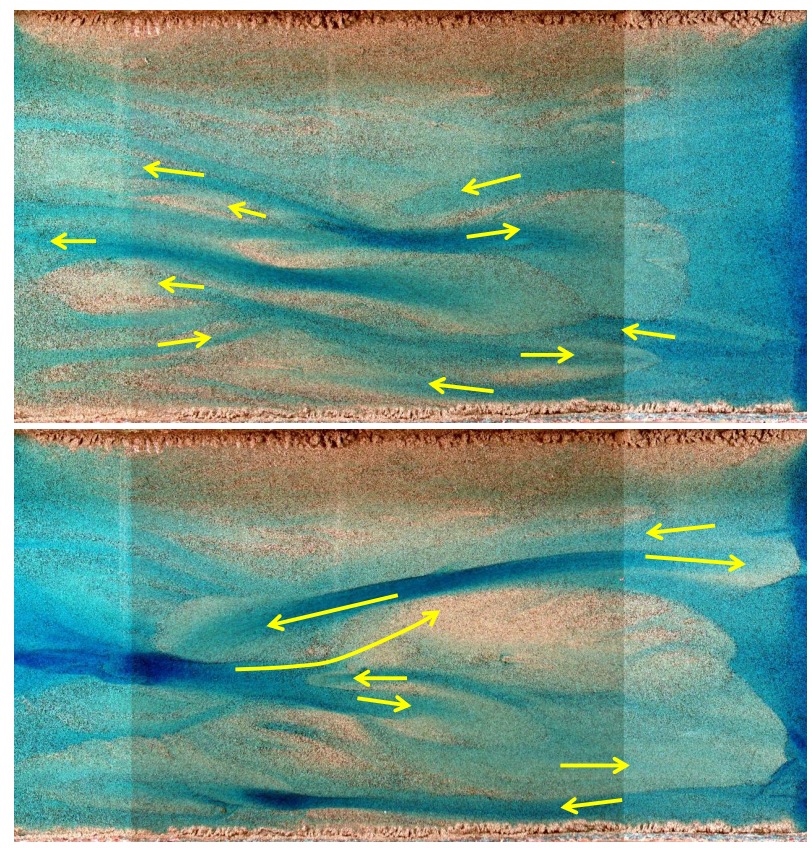

Fig. 3. Top view on transitional V-shaped to diamond-shaped tidal bars in moderate-energy experiment 2 after about 300 and 3000 tidal cycles. Arrows indicate dominant tidal flow direction.

diamond shape arose as a consequence of the flow in opposite direction bifurcating around the high lobate bar head and eroding the head somewhat into a blunt V-shape. All bars grew further by bar merging and bar overtaking.

Mid-channel bars with somewhat symmetrical bifurcations such as found in braided systems were rare. This is confirmed by our control experiments of braiding that were dominated by mid-channel bars and lateral accretion bars and had a very different overall appearance.

After about 4000-6000 tidal cycles (about $100 \mathrm{~h}$ ) the experiments were in dynamic equilibrium in the sense that the overall pattern no longer changed much and the number of bars had stabilized by merging of smaller bars. At that point the sediment was still mobile but bars hardly migrated anymore. The more energetic experiments were not much faster because the bars developing there were larger and required more formation time.

We tested several possible relations between boundary conditions and bar dimensions. Average bar length was about $1 / 8$ of the tidal excursion length. The correlation was $R^{2}=0.83$ for $n=8$ experiments, significant above the $p=$ 0.05 level. Furthermore we observed a correlation of similar strength between bar height and bar width, which have a typical ratio of $1 / 40$. Surprisingly, however, we observed no significant correlations between bar length and width or height, or between the tidal excursion length and bar width or height.
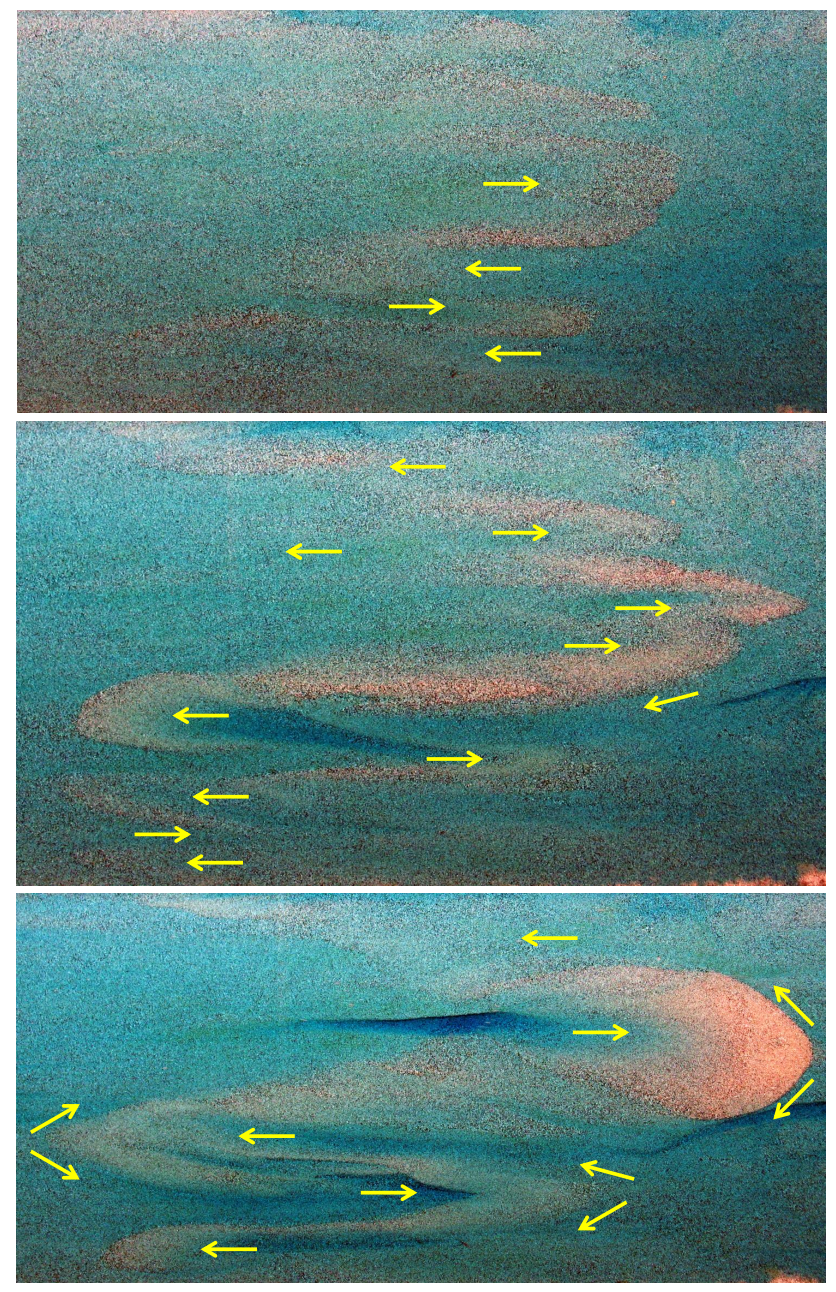

Fig. 4. Top view on transitional V-shaped to diamond-shaped tidal bars in moderate-energy experiment 3 after about 200, 1000 and 2000 cycles.

\section{Discussion}

The present experiments represent tidal bars in a short reach of an estuary and were done with exactly symmetrical conditions and two open boundaries. The experiments of Tambroni et al. (2005) in a much longer flume had one open boundary. A short reach in their flume showed alternate tidal bars, in contrast to our short reach which had many parallel bars and channels and can be seen as a form of tidal braiding. The difference is that the Tambroni et al. experiments have a channel width-to-depth ratio of about 4 , just above the threshold for alternate bar formation (Seminara and Tubino, 2001) whereas our width-to-depth ratio is about 200, which is much closer to natural values for, e.g., the Western Scheldt estuary which has a width of roughly $5 \mathrm{~km}$ and a depth of the order of $10 \mathrm{~m}$. In rivers such a width-to-depth ratio would certainly lead to braiding (Kleinhans and van den Berg, 2011). 

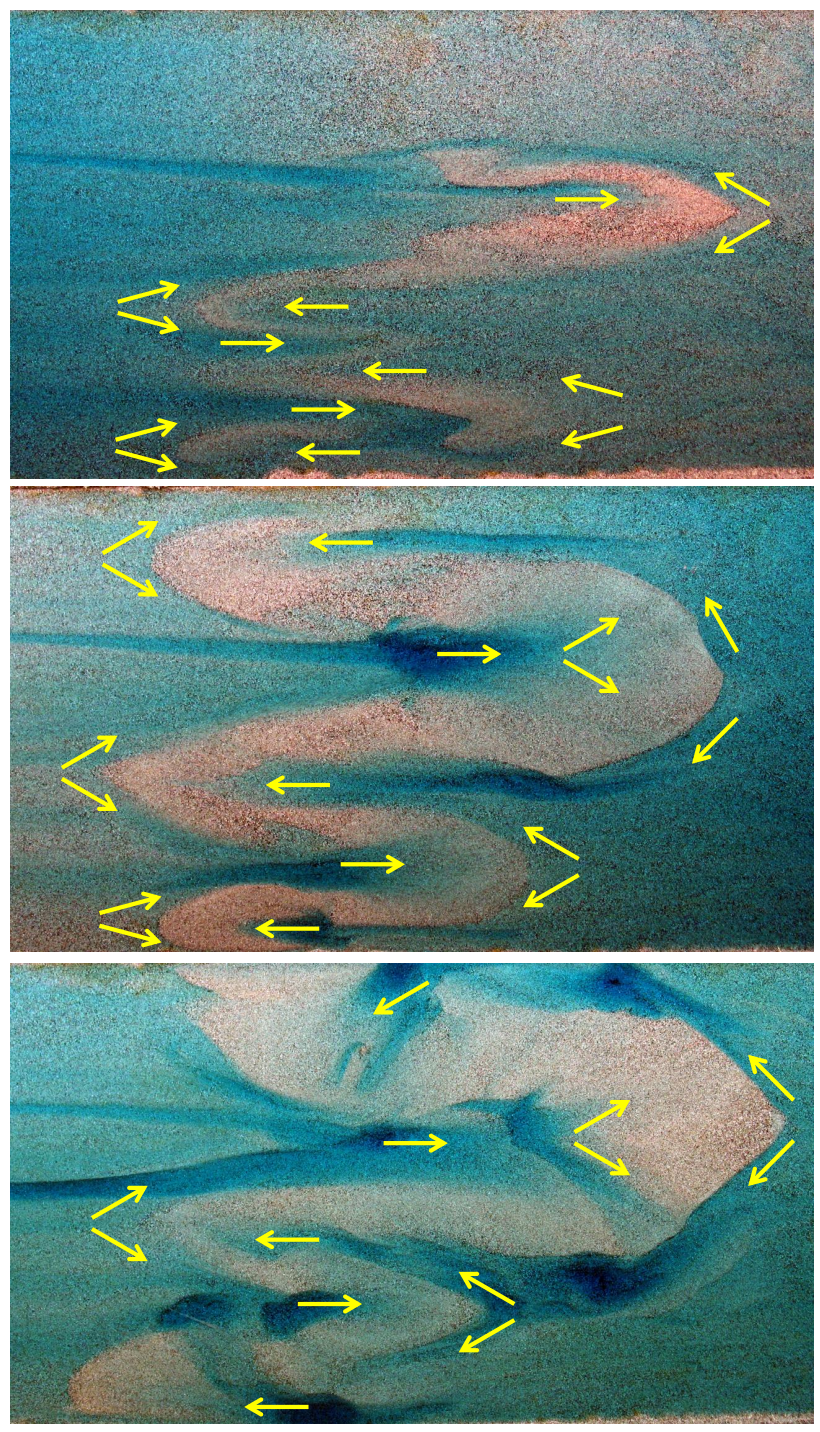

Fig. 5. Top view on diamond-shaped tidal bars in high-energy experiment 4 after about 500, 1000 and 3000 cycles.

The diamond shaped bars formed as a result of flow bifurcating around the bar head during the opposite tidal phase. The formation of this blunt V-shape is reminiscent of formation of bifurcations in braided rivers (Federici and Paola, 2003). Perhaps also the V-shape of the incipient bars is due to such bifurcation effects. The analogy raises the question to what extent tidal bifurcations with mutually evasive ebband flood-dominated channels form by similar processes of flow and sediment partitioning, transverse bed slope effects and helical flow due to channel curvature (Kleinhans et al., 2013).

In all experiments relatively deep scour holes emerged and it is not clear to what extent these are a combination of artifacts or scours associated to confluences. The scour holes possibly arise due to the hydraulic smooth boundary caus- ing spatial laminar to turbulent boundary layer transitions Van Dijk et al. (2012). The tendency to scour was stronger in higher-energy experiments where bars became larger.

The observed relation between tidal excursion length and bar wavelength qualitatively confirms the bar theory of Schramkowski et al. (2002). However, this requires further investigation, because the tidal excursion length itself is of similar length as the entire flume. Furthermore it is well possible that the channel width-to-depth ratio also determines bar length, and both theories could be valid in different conditions, which we have not tested yet.

The present experiments allowed testing in smaller channel width-to-depth ratios than reported in earlier experiments, particularly Tambroni et al. (2005). The reason is that earlier experiments require sufficient water depth to develop enough shear stress for sediment mobilisation, whereas the novel tilting flume setup causes mobilisation by tilting which works at any water depth. As such the relatively low widthto-depth ratio in Tambroni et al. can be seen as a scale effect. In the tilting flume, any width can be specified, and, if processes that form mud flats and tidal marshes can be simulated, perhaps system width can also be formed as part of the processes rather than imposed as an initial condition.

These experiments together show that such tidal experiments are feasible and useful, but that a larger facility is required for more reliable scaling of natural morphology. In short, our work is evidence that experimental creation of tidal systems is possible in a fast tilting flume setup and inspiration for further development of tidal tilting flume experiments.

\section{Conclusions}

Tidal bars formed in an experimental facility where flume tilting caused periodically reversing tidal flow and sediment transport. The incipient bars have a sharp V-shape connected in an alternating saw-tooth pattern. Larger bars are wider and more lobate and often develop blunt V-shaped heads as the flow in opposing direction bifurcates around the bar. As a result, a system evolved where some parallel channels and bars were nearly entirely flood-dominated and others nearly entirely ebb-dominated in agreement with observations in nature. These mutually evasive ebb- or flood-dominated channels probably arise from a combination of flow evading the steep heads of incipient, asymmetrical bars formed by the flow in opposite direction.

Acknowledgements. We cordially thank Henk Markies and Marcel van Maarseveen for design and technical support, and Renske Terwisscha van Scheltinga, Howard Feldman, Jim Best and Chris Paola for discussion. Reviews by Zhou Zeng and Stefano Lanzoni and guidance by Giovanni Coco are gratefully acknowledged. 


\section{References}

Dalrymple, R. and Rhodes, R.: Estuarine dunes and bars, in: Geomorphology and sedimentology of estuaries, edited by: Perillo, G. 359-422, Elsevier Science, New York, 1995.

Federici, B. and Paola, C.: Dynamics of channel bifurcations in noncohesive sediments, Water Resour. Res., 39, 1162, doi:10.1029/2002WR001434, 2003.

Hibma, A., Schuttelaars, H. M., and Wang, Z. B.: Comparison of longitudinal equilibrium profiles of estuaries in idealized and process-based model, Ocean Dynam., 53, 252-269, 2003.

Kleinhans, M. G. and van den Berg, J. H.: River channel and bar patterns explained and predicted by an empirical and a physicsbased method, Earth Surf. Process. Landforms, 36, 721-738, doi:10.1002/esp.2090, 2011.

Kleinhans, M. G., van der Vegt, M., Terwisscha van Scheltinga, R., Baar, A., and Markies, H.: Turning the tide: experimental creation of tidal channel networks and ebb deltas, Netherlands $\mathbf{J}$. Geosci., 91, 311-323, 2012.

Kleinhans, M. G., Ferguson, R. I., Lane, S. N., and Hardy, R. J.: Splitting rivers at their seams: bifurcations and avulsion, Earth Surf. Process. Landforms, 38, 47-61, doi:10.1002/esp.3268, 2013.

Mayor-Mora, R.: Laboratory Investigation of Tidal Inlets on Sandy Coasts, Univ. of California, Berkeley, Hydr. Eng. Lab., 1977.
Reynolds, O.: Report of the committee appointed to investigate the action of waves and currents on the beds and foreshores of estuaries by means of working models, British Association Report, in: Papers on mechanical and physical subjects, Vol. II 1881-1900, Cambridge Univ. Press, 1901, Chapter 57, 380-409, 1889.

Schramkowski, G. P., Schuttelaars, H. M., and de Swart, H. E.: The effect of geometry and bottom friction on local bed forms in a tidal embayment, Cont. Shelf Res., 22, 1821-1833, 2002.

Seminara, G. and Tubino, M.: Sand bars in tidal channels. Part 1. Free bars, J. Fluid Mech., 440, 49-74, 2001.

Stefanon, L., Carniello, L., D’Alpaos, A., and Lanzoni, S.: Experimental analysis of tidal network growth and development, Cont Shelf Res., 30, 950-962, doi:10.1016/j.csr.2009.08.018, 2010.

Tambroni, N., Bolla Pittaluga, M., and Seminara, G.: Laboratory observations of the morphodynamic evolution of tidal channels and tidal inlets, J. Geophys. Res., 110, F04009, doi:10.1029/2004JF000243, 2005.

Van Dijk, W. M., Van de Lageweg, W. I., and Kleinhans, M. G.: Experimental meandering river with chute cutoffs, J. Geophys. Res., 117, F03023, doi:10.1029/2011JF002314, 2012.

van Veen, J.: Ebb and flood channel systems in the Netherlands tidal waters, Journal of the Royal Dutch Geographical Society, 67, 303-325, 1950. 